\title{
VLBI ASTROMETRY OF RADIO-EMITTING STARS
}

\author{
J.-F. LESTRADE \\ Observatoire de Paris-Meudon, arpeges ura-cnrs 1757 \\ F92195 - Meudon - Principal Cedex - France \\ D.L. JONES, R.A. PRESTON \\ Jet Propulsion Laboratory, Calfornia Institute od Technology \\ 4800 Oak Grove, Pasadena, CA, 91109, USA \\ R.B. PHILLIPS
}

Haystack Observatory, Massachussets Institute of Technology Westford, Mass, 01886, USA

AND

J. KOVALEVSKY, M. FROESCHLE, F. MIGNARD

Centre d'Etudes et de Recherche en Géodynamique et Astronomie F06130 - Grasse - France

\begin{abstract}
High-accuracy astrometric VLBI observations of 7 radio stars are presented with applications to the connection between the Hipparcos and VLBI extragalactic reference frames, to the identification of the radio emitting region in the ternary system Algol and the detectabity of Jupitersize planet orbiting the radio star $\sigma^{2} \mathrm{CrB}$.
\end{abstract}

\section{Introduction}

Very Long Baseline Interferometry (VLBI) observations of radio-emitting stars conducted over the last decade have yielded their positions, annual proper motions, trigonometric parallaxes with a precision of 1 milliarcsec (mas) or better. The applications of such high-accuracy astrometric parameters include the improvement of the astronomical distance scale through trigonometric parallax measurements, the connection of the radio and optical radio celestial reference frames, the attempt to detect Jupiter-size companions orbiting radio-emitting stars, the determination of the orientation (node) and angular separation of binary system orbits for dynamical studies of these systems, the determination of the crucial proper motion of 


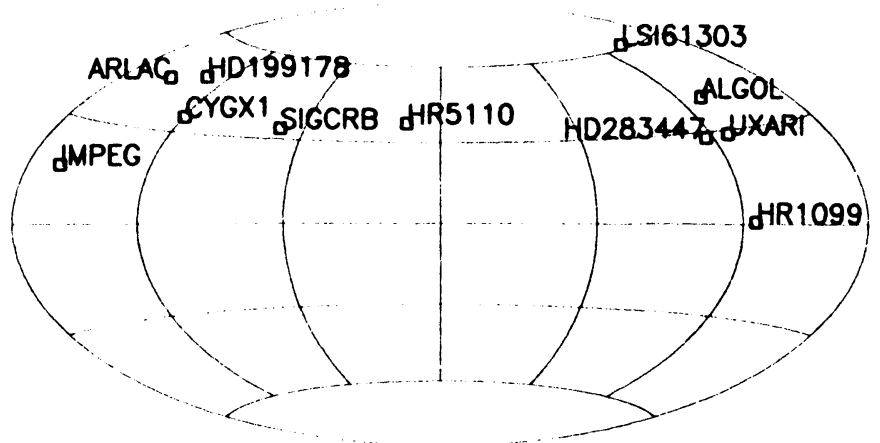

Figure 1. Sky distribution of the VLBI link stars for Hipparcos.

the reference star in the Gravity Probe B mission to test the Lense-Thirring effect of General Relativity.

There are about 30 stars detectable with the state of the art VLBI data acquisition system and there might be 100 detectable by the end of the decade with the new VLBI capabilities being developed in the US and in Europe.

Our VLBI astrometric program of 11 radio-emitting stars (Figure 1) started in 1982 and its initial motivation was to connect the Hipparcos optical reference frame to the VLBI (radio) extragalactic reference frame at the milliarcsec level. The flux densities of these 11 stars are variable between a few milliJansky and a few tens milliJansky, i.e. 100 to 1000 times weaker than the extragalactic radio sources usually observed by VLBI. We had to resort to the phase-referencing VLBI technique both to enhance sensitivity with multi-hour integrations and to achieve high astrometric accuracy through use of the differential inteferometric phase between the target star and an angularly nearby VLBI extragalactic source. The details of this technique are described in Lestrade et al (1990). We shall summarise the state of the Hipparcos/VLBI connection based on our program and present two additional results as spin-offs of these VLBI observations.

\section{Preliminary link of the Hipparcos and VLBI reference frames}

We have carried out a comparison between the VLBI and Hipparcos astrometric parameters of seven radio-emitting stars of our program. Both techniques (VLBI and Hipparcos) have provided positions, annual proper motions and trigonometric parallaxes of these stars with comparable formal uncertainties at the 1 mas level or better. We have found that the systematic discrepancies between these two sets of astrometric parameters can be 
removed by performing a single global rotation between the extragalactic and Hipparcos reference frames. The three angles and annual rates of this rotation are determined at better than the milliarcsec level as shown in Table 1. The Hipparcos parameters used for this determination are from the Hipparcos reduction consortium FAST but a similar result has been found with the other consortium NDAC.

TABLE 1. VLBI/Hipparcos-FAST connection: the 3 angles $\left(A_{1}\right.$, $\left.A_{2}, A_{3}\right)$ and the 3 rates of rotation $\left(\dot{A}_{1}, \dot{A}_{2}, \dot{A}_{3}\right)$ between the Hipparcos and VLBI reference frames have been determined with 7 link stars.

$$
\begin{aligned}
& \text { Weighted Least-squares-fit solution } \\
& \text { Rotation angles at epoch } 1991 \mathrm{Apr} \\
& A_{1}=-26.87 \pm 0.46 \mathrm{mas} \\
& A_{2}=-12.64 \pm 0.55 \mathrm{mas} \\
& A_{3}=22.99 \pm 0.46 \mathrm{mas} \\
& \text { Rotation rates }: \\
& \dot{A}_{1}=0.60 \pm 0.48 \mathrm{mas} / \mathrm{yr} \\
& \dot{A}_{2}=0.06 \pm 0.46 \mathrm{mas} / \mathrm{yr} \\
& \dot{A}_{3}=1.35 \pm 0.43 \mathrm{mas} / \mathrm{yr}
\end{aligned}
$$$$
\text { Rotation angles at epoch } 1991 \text { April } 1 \text { : }
$$

The robustness of the solution has been tested by splitting the 7 FAST link stars into two independent subsets, one with 3 stars (HR1099, HR5110, AR Lac) and one with 4 stars (UX Ari, $\sigma^{2}$ CrB, CygX1, IM Peg). Rotation angles and rates have been solved independently for with these two subsets and compared. The differences are no more than the quadratically combined uncertainties of the two solutions (Table 2).

The three angles of rotation are consistent with the expected values since the FAST Hipparcos coordinate system is aligned upon a quasi-FK5 reference frame and the FK5 is aligned with the VLBI reference frame to within 70 mas. The measured rotation rates are consistent with the uncertainty of about $0.5 \mathrm{mas} / \mathrm{yr}$ in Table 1 , although the $\dot{A}_{3}$ rate reaches $3 \sigma$. This means that the procedure used in the Hipparcos data reduction to stop the rotation of the Hipparcos sphere is efficient.

The angles of rotation found are relative to a VLBI extragalactic reference frame that is defined by the International Earth Rotation Service (IERS) VLBI coordinates of the extragalactic reference sources (Arias, Feissel, Lestrade 1991) used for the differential VLBI measurement of the link stars. The post-fit residuals of the star coordinates and proper motion com- 
TABLE 2. Differences between the angles and rates of rotation determined after splitting the 7 VLBI link stars into two independent subsets of 3 and 4 stars. The symbol $\sigma$ is the quadratically combined uncertainties of the 2 solutions.

\begin{tabular}{ll}
\hline $\begin{array}{l}\text { Rotation } \\
\text { parameters }\end{array}$ & $\begin{array}{l}\text { Differences between solutions } \\
{[\mathrm{mas}]}\end{array}$ \\
\hline$A_{1}$ & $+0.53 \sim 0.5 \sigma$ \\
$A_{2}$ & $-0.48 \sim 0.5 \sigma$ \\
$A_{3}$ & $+0.08 \sim 0.1 \sigma$ \\
$\dot{A}_{1}$ & $+1.17 \sim 1.0 \sigma$ \\
$\dot{A}_{2}$ & $+0.91 \sim 1.0 \sigma$ \\
$\dot{A}_{3}$ & $+0.54 \sim 0.5 \sigma$ \\
\hline
\end{tabular}

ponents after the adjustment of the global rotation indicate that the consistency between the Hipparcos and VLBI astrometric techniques is at the milliarcsec level. This is the first cross-check between these two astrometric techniques of comparable precision. The level of agreement found is consistent with the expected accuracy of the technique, even though the astrometric parameters used are from preliminary reductions of the data for both techniques. A complete account of this preliminary link is in Lestrade et al (1994a).

\section{VLBI Astrometric Identification of the Radio-emitting Region in Algol}

Spectroscopy, photometry and speckle interferometry have already provided accurate values for almost all the geometric and orbital parameters of the ternary system Algol (e.g. Söderhjelm 1980). Consequently, it is known that the mass ratio $M_{A} / M_{B}$ between the primary (main-sequence) and secondary (subgiant) stars of the close binary of this system is $\sim 4.5$ and thus significantly different from unity. This makes the motion of the radio source quite different depending on whether it is associated with the subgiant or the main sequence star of the close-binary (astrometric measurements exclude that the third component of the system could be responsible for the radio emission). The maximum displacement of the radio source projected on the sky occurs between the quadrature points of the close binary orbit. This means between orbital phase $\phi=0.25$ and $\phi=0.75$, if $\phi=0$ is the primary eclipse time when the cool subgiant is in front of the bright B8V star. The magnitude of this displacement would be $0.0036^{\prime \prime}$ (from $\left.2 \times \frac{M_{A}}{M_{A}+M_{B}} \times a\right)$ if the emitting region is centered on the subgiant but it 


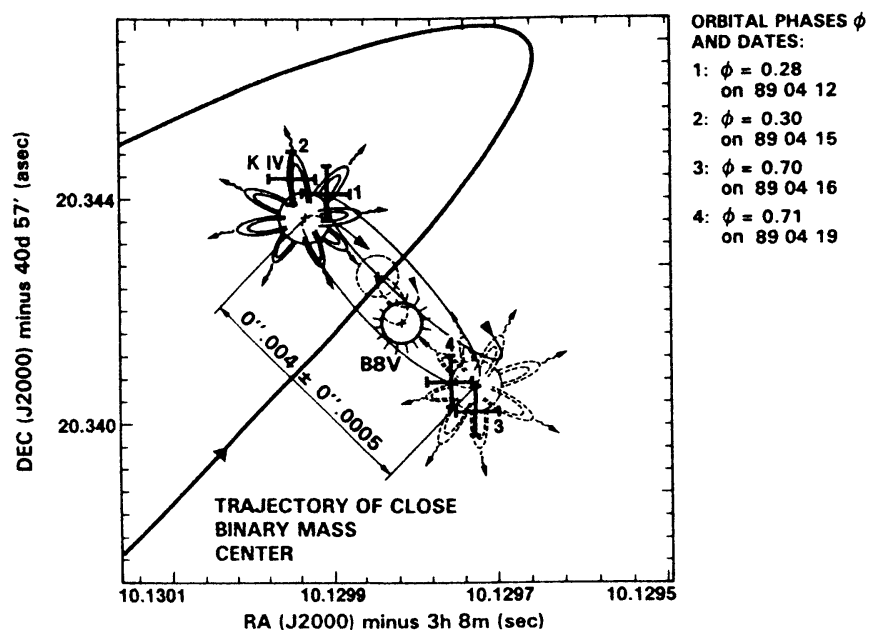

Figure 2. The four positions of the radio emission of Algol (crosses) measured by VLBI are superposed on the configuration of the close binary during the observations at orbital phases 0.25 and 0.75 . A portion of the trajectory of the close binary mass center orbiting the third star in $1.86 \mathrm{yr}$ is displayed.

would be $0.0008^{\prime \prime}$ (from $2 \times \frac{M_{B}}{M_{A}+M_{B}} \times a$ ) if centered on the more massive main sequence star B8. $a$ is the semi-major axis and is known. The displacement would be intermediate if the emitting region is associated with the transient flow of gas between the two stars.

In April 1989, we made four VLBI astrometric observations of the Algol radio emission. The observations took place on April 12th, 15th, 16th and 19 th, allowing 2.5 orbital revolutions of the close binary to be sampled over these 7.5 days. These four epochs were chosen so that the observations took place when the two stars of the close binary were as close as possible to the orbital phases $\phi=0.25$ and $\phi=0.75$. A single observation at each of these two orbital phases would have sufficed, but two additional observations were scheduled because of possible technical failures during the observations and for redundancy of the measurement. All four observations were successful.

We measured the displacement of the radio source in Algol of $4 \pm 0.5$ mas between two orbital phases 0.25 and 0.75 over two consecutive orbital revolutions of the close binary (see Figure 2). The magnitude of the displacement unambiguously indicates that the less massive star of the close binary, the $\mathrm{K}$ subgiant, is the star responsible for the non-thermal radio emission of the system. This is consistent with the idea that the radio emission in Algol is related to the strong magnetic activity of the subgiant.

The orientation and sense of the displacement on the sky that are di- 


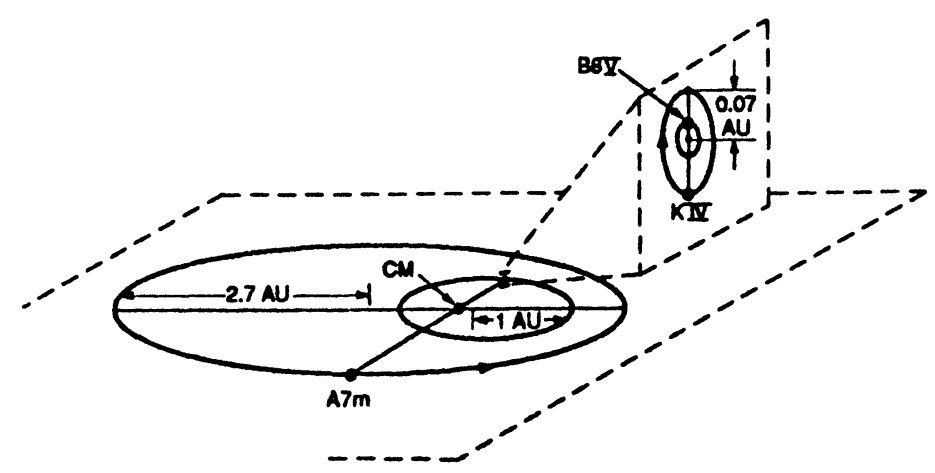

Figure 3. Configuration of the two orbital planes in the Algol ternary system.

rectly deduced from our astrometric VLBI observations imply 1) that the orbital plane of the close binary is at p.a. $=+52 \pm 5^{\circ}$ (p.a. $=0^{\circ}$ is North and p.a. $=90^{\circ}$ is East) and 2) that the sense of circulation of the close binary is clockwise, as seen on the sky. Thus, the long-period and close binary orbital motions are almost orthogonal and counter-revolving (Figure 3). The orientation and sense of circulation of the close binary determined by our VLBI observations are identical to the findings of Rudy (1979) and Kemp et al $(1981,1983)$ obtained less directly with optical polarisation observations. This intriguing configuration is difficult to reconcile with our present idea on the formation, evolution and dynamical stability of multiple stellar systems, since it is difficult to conceive that the angular momentum of the primordial cloud of this ternary system was split along two orthogonal axis. For a full account of these observations see Lestrade et al 1993.

\section{Astrometric detectability of a Jupiter-size planet orbiting the radio-emitting star $\sigma^{2} \mathbf{C r B}$}

The motion of a single planet in a circular orbit around a star causes the star to undergo a reflexive circular motion around the star-planet barycenter. When projected on the sky, the orbit of the star appears as an ellipse with angular semimajor axis $\theta$ given by $\theta=\frac{m_{p}}{M_{*}} \frac{a}{d} \quad(e q .1)$ where $\theta$ is in $\operatorname{arcsec}$ when the semimajor axis $a$ is in AU, the mass of the planet $\left(m_{p}\right)$ and the mass of the star $\left(M_{*}\right)$ are in solar masses and the distance $d$ is in pc. For example, observing the solar system from a distance of $10 \mathrm{pc}$, the presence of Jupiter would be revealed as a periodic circular displacement in the Sun's position, with a diameter $2 \theta$ of 1.0 mas and a period of 11.9 years.

Ground-based optical astrometry has generally been limited to a precision of a few tens of milliarcsec although the best measurements are at the 1 mas level now (Gatewood et al 1992). VLBI astrometric observations of 


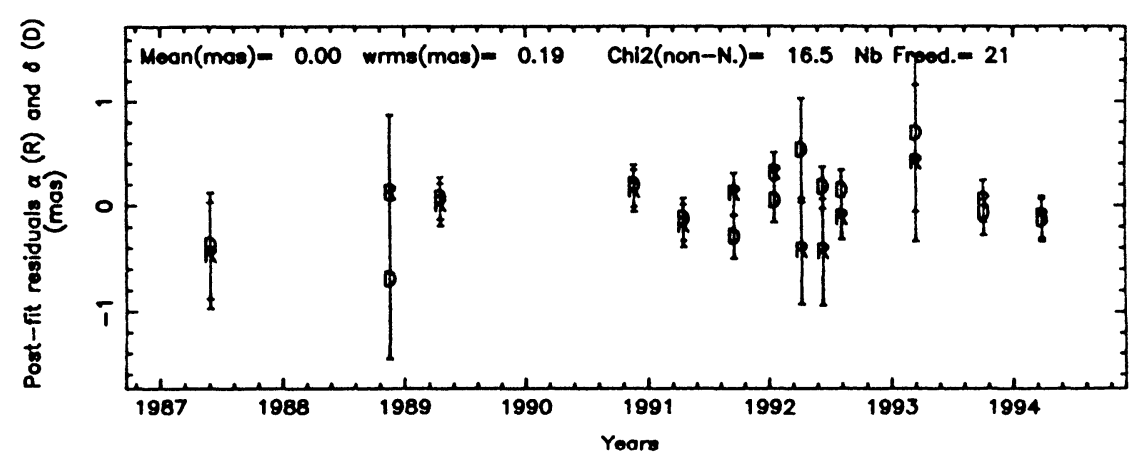

Figure 4. Post-fit coordinate residuals for $\sigma^{2} \mathrm{CrB}$. The 5 astrometric parameters of $\sigma^{2} \mathrm{CrB}$ (coordinates, proper motion components and trigonometric parallax) are adjusted to the coordinates measured by VLBI at 13 epochs.

$\sigma^{2} \mathrm{CrB}$ conducted by us at 13 epochs between 1987 and 1994 have yielded its position, proper motion and trigonometric parallax and the resulting post-fit coordinate residuals are characterised by an $\mathrm{rms}$ as small as 0.2 mas (see Figure 4). The formal uncertainties for the 5 fitted astrometric parameters are 0.08 mas for the relative position between $\sigma^{2} \mathrm{CrB}$ and the reference source $1611+343,0.04$ mas/year for the proper motion and 0.08 mas for the trigonometric parallax (Lestrade et al 1992, 1994b).

The lack of a clear sinusoidal signature in the post-fit coordinates residuals of Figure 4 sets a limit on the presence of a planet around $\sigma^{2} \mathrm{CrB}$ for the 7 years observation span. The rms of the post-fit residuals $(0.2$ mas in Figure 4) is an upper limit on any systematic departure from linear motion of the star. Eq (1) can be used to exclude a range of planetary perturbations by taking $2 \theta=0.2$ mas, $M_{*}=2.26 M_{\odot}$ and d $=22.7 \mathrm{pc}$ for $\sigma^{2} \mathrm{CrB}$. The log-log representation of eq (1) with these parameters is in Figure 5 and in Lestrade et al (1994b). The diagonal line of constant astrometric signature in Figure 5 follows eq (1) for $2 \theta=0.2$ mas. We assume that a full orbital period of the planet must be sampled during the observations to separate the sinusoidal planetary signature from the fitted linear proper motion. In these conditions, the maximum semimajor axis $a$ of the orbit of a planet corresponds to the total observation span through the third Kepler law. This lower limit on $a$ is $4.8 \mathrm{AU}$ for the 7 years of observations and is indicated by the vertical dashed line in Figure 5 . Finally, the shaded area indicates the planet parameter space $\left(a, m_{p}\right)$ that are excluded by the present observations, i.e. any planetary perturbations in the shaded area is too large to be consistent with the post-fit coordinate residual rms of 0.2 mas measured over 7 years by VLBI. Note that for $a=4.8 \mathrm{AU}$, the upper limit on the planet mass $m_{p}$ is $0.0012 M_{\odot},(\sim 1$ Jupiter mass $)$ as derived 


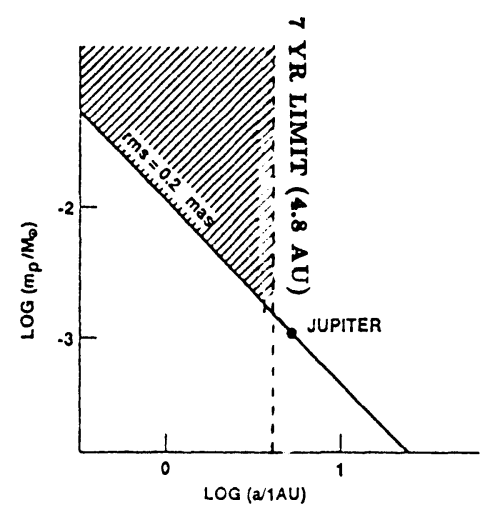

Figure 5. Log-Log representation of eq (1) for the rms of the post-fit coordinate residuals $\left(0.2\right.$ mas) of $\sigma^{2} \mathrm{CrB}$. The shaded area is the parameter space (semi-major axis $a$, mass $m_{p}$ ) that are excluded by our 7 years of VLBI observations for a planet around this star.

by eq.(1) for $\sigma^{2} \mathrm{CrB}$ and $2 \theta=0.2$ mas.

This limit will become more stringent if the period of observation is extended. The ultimate goal of our program is now to extend these 7 years of astrometric VLBI observations of $\sigma^{2} \mathrm{CrB}$ to complete a full 12 year cycle typical of a Jupiter-size planet that might orbit this star. The goal is also to improve the level of precision of our astrometric measurement since this level is not presently SNR-limited. It might reach 20 microarcseconds if all systematic errors could be removed by an improved strategy of observations and data analysis.

\section{References}

Arias, F., Feissel, M., Lestrade, J-F., 1991, The IERS extragalactic Reference Frame and its Tie to Hipparcos, IERS Technical Note 7, December 1991, Observatoire de Paris, France.

Gatewood, G., Stein, J., Joost Kiewiet de Jonge, T., Persinger T., Reiland, T., 1992, Astron. J., 104, 1237

Lestrade J.-F., Rogers A.E.E., Whitney A.R., Niell A.E., Phillips R.B., Preston R.A., 1990, Astron. J., 99, 1663-1673.

Lestrade J.-F., Phillips R.B., Preston, R.A., D. C. Gabuzda, 1992, Astron. Astroph., 258, $112-115$

Lestrade J.-F., Phillips R.B., Hodges, M.W., Preston, R.A., 1993, Astroph. J., 410, 808814.

Lestrade J.-F., Jones, D.L., Preston, R.A., Phillips R.B., Titus M.A., Kovalevsky J., Lindegren L., Hering R., Froeschle M., Falin, J-L, Mignard, F., Jacobs, C.S., Sovers, O.J., Eubanks, M., Gabuzda, D., 1994a, Astron. Astroph. (submitted)

Lestrade J.-F., Jones, D.L., Preston, R.A., Phillips R.B., 1994b, Astroph. and Space Science, vol. 212, Kluwer Academic Publishers, p.251-260.

Rudy, R.J., 1979, MNRAS, 186, 473

Kemp, J.C., Barbour, M.S., McBinney, R.E., Rudy, R.J., 1981, Astroph. J., 243, 557

Söderhjelm, S., 1980, Astron. Astroph., 89, 100. 